\title{
Dual processes in recognition: Does a focus on measurement operations provide a sufficient foundation?
}

\author{
MICHAEL S. HUMPHREYS, SIMON DENNIS, \\ KERRY A. CHALMERS, and SIMON FINNIGAN \\ University of Queensland, Brisbane, Queensland, Australia
}

\begin{abstract}
Current theoretical thinking about dual processes in recognition relies heavily on the measurement operations embodied within the process dissociation procedure. We critically evaluate the ability of this procedure to support this theoretical enterprise. We show that there are alternative processes that would produce a rough invariance in familiarity (a key prediction of the dual-processing approach) and that the process dissociation procedure does not have the power to differentiate between these alternative possibilities. We also show that attempts to relate parameters estimated by the process dissociation procedure to subjective reports (remember-know judgments) cannot differentiate between alternative dual-processing models and that there are problems with some of the historical evidence and with obtaining converging evidence. Our conclusion is that more specific theories incorporating ideas about representation and process are required.
\end{abstract}

From almost the earliest days of experimental work on human memory, recognition has been acknowledged as being in some sense "easier" or "simpler" than recall. In order to explain this greater simplicity, McDougall (1904) proposed that a correct response required a lower threshold for recognition than for recall. An alternative, proposed by James (1890), was that recall involved a search process that was not required for recognition.

Kintsch (1970) provided a more contemporary version of James's (1890) proposal. According to Kintsch, in recognition, the to-be-recognized item provided direct access to the memory trace, whereas recall involved a trace to trace search process. Not only did this proposal explain the greater "simplicity" of recognition but it appeared to provide a means of distinguishing storage and retrieval processes. That is, if Kintsch were correct, a variable that affected free recall but not single-item recognition could be assumed to affect retrieval but not storage. Kintsch's proposal was based on a literature review that seemed to show that "organization" (e.g., a comparison between a study list where there are no obvious relationships among the words and a list where there are several items from each of a small number of taxonomic categories) affected recall but not recognition.

Several lines of evidence were soon advanced contradicting Kintsch's (1970) conclusion that organizational

This work was supported by Grant A79701517 from the Australian Research Council to the first author. The authors thank Charles Brainerd and Elliot Hirshman for their helpful comments on an earlier draft of this article. Correspondence should be addressed to M. S. Humphreys, The ARC Key Centre for Human Factors and Applied Cognitive Psychology, University of Queensland, Brisbane, QLD 4072, Australia (email: m.humphreys@humanfactors.uq.edu.au). manipulations did not affect single-item recognition. Atkinson and Juola (1974) summarized empirical and theoretical developments from a series of papers showing that recognition latencies increased with the length of a well-learned list (interpreted as evidence for a search process plus a rapid direct access process: familiarity). Mandler (1972) also reviewed evidence from several studies in which subjects were first required to sort a list of words with little or no normative organization into a set of subjective or personal categories. Under these conditions, the probability of correctly recognizing old words, like the probability of recall, tended to increase with increases in the number of subjective categories used by a subject. Other data showed that blocking of items by normative category in the study list improved recognition accuracy, even when the blocked items were not tested together, and that the recognition of one word that had been studied as part of a pair was better in the context of the pair member than in any other context. The previously mentioned work is summarized in Mandler (1980) and Humphreys and Bain (1983). This work produced a substantial amount of evidence in support of the hypothesis that two processes or two types of information were being used in recognition. Humphreys and Bain, however, were able to show that most of this evidence either did not or could not in principle differentiate between Mandler's independence assumption (two processes, familiarity and recall, are assumed to be independent) and Humphreys's (1976) redundancy assumption (relational information is assumed to be unavailable unless item information about the cue is available).

Following the Humphreys and Bain (1983) and Mandier (1980) reviews, much of the empirical and theoretical work on dual processes in recognition was focused on 
Jacoby and Dallas's (1981) proposal that familiarity (the simpler of the two hypothesized recognition components) was the same as perceptual fluency (the priming effect that is observed with implicit memory instructions). This was a highly testable proposal where it would have been possible to produce definitive support. However, definitive support was not forthcoming, and many of the parallels that were first drawn between familiarity and perceptual fluency have been shown not to hold (Chalmers \& Humphreys, 1998; Knowlton, 1998; Poldrack \& Logan, 1997).

As interest in establishing a link between perceptual fluency and familiarity has waned, the process dissociation procedure developed by Jacoby (1991) has come to play a dominant role in dual-process theory. This procedure is based on a measurement model, and the measurement operations embodied in this model are driving theory development. In this paper, we examine some of the problems that occur in this approach to theory development.

\section{Dual Processing and the \\ Process Dissociation Procedure}

Current theoretical thinking about dual processes in recognition hinges on the distinction between automatic and controlled processes (sometimes denoted "conscious" and "unconscious" processes, "intentional" and "unintentional" processes, or "aware" and "unaware" processes). Automatic processes are assumed to produce a feeling of familiarity that, in the context of episodic recognition, tends to evoke an "old" response, independent of subject control. Familiarity is often considered to be the same construct that underlies perceptual fluency. In contrast, controlled processes are assumed to be based on recollection - retrieving some aspect of the study opportunity that can be used to infer that the item appeared in the list (i.e., "I remember solving an anagram for this item and I only solved anagrams in the study list, so this item must have been in the study list"). This source of information is assumed to be subject to control by the participant and to be subject to interference by concurrent activity, and it is generally assumed to be open to awareness.

To separate the influences of familiarity $(F)$ and recollection $(R)$ in episodic recognition, Jacoby (1991) devised the process dissociation procedure. In a typical application of this procedure, subjects study two lists and are asked to make one of two recognition decisions at test. In the inclusion condition, they are required to respond "old" if the item was in either of the two lists. In the exclusion condition, they are required to respond "old" only if the item appeared in the target list (either List 1 or List 2 ) and not in the nontarget list. The probability of a correct "old" response in the inclusion condition is represented as $I$, and the probability of incorrect "old" response to a nontarget "old" item in the exclusion condition is represented as $E$. In a variation on this basic design, Yonelinas (1994) had subjects perform just the second task (i.e., say "old" only if the item appeared in the target list) and defined $I$ as the probability that the subject said "old" to a word in the target list and $E$ as the probability that a subject said "old" to an item in the nontarget list.

Jacoby (1991) assumes that, in the inclusion condition, the subject will respond "old" if the item is familiar or if it is recollected:

$$
I=F+R-F R .
$$

In the exclusion condition, when the subject should be responding "new," it is assumed that they will mistakenly respond "old" if the item is familiar but they fail to recollect which list it occurred in:

$$
E=F(1-R) .
$$

These equations can be solved for $R$ and $F$, giving:

$$
R=I-E
$$

and

$$
F=E /(1-I+E) .
$$

The process dissociation procedure and the theory that is intertwined with the procedure have no representational assumptions and have a minimal commitment to process details. ${ }^{1}$ This lack of specificity about representation and process also means that measurement operations (in this case, embedded within a measurement model) take priority in theory development. As a consequence, support for the theory critically depends on the assumptions underpinning the measurement model-in particular, the assumption that $F$ and $R$ are independent. One challenge to this assumption has come from demonstrations that changes in list discriminibility (are the two lists processed in similar or dissimilar ways, or are the features that the subject is required to discriminate well or poorly learned?) can produce tradeoffs between the estimates of $F$ and $R$. The implications of this tradeoff have been discussed elsewhere (Dodson \& Johnson, 1996; Gruppuso, Lindsay, \& Kelley, 1997; Mulligan \& Hirshman, 1997; Yonelinas \& Jacoby, 1996). In this paper, we will first consider the evidence provided by findings that some experimental manipulations change estimates of $R$ while leaving estimates of $F$ unchanged. These demonstrations of the invariance of $F$ are central to the distinction between automatic and controlled processes and the assumed independence of $F$ and $R$. If the theoretical enterprise that depends on these assumptions is to be accepted, it is necessary to show that alternative explanations for the invariance of $F$ can be rejected.

\section{The Invariance of $\boldsymbol{F}$}

Included among the variables that have been examined using the process dissociation procedure are (1) generate versus read (Jacoby, 1991), (2) aging (Jennings \& Jacoby, 1993), (3) list length (Yonelinas \& Jacoby, 1994), (4) duration of study (Yonelinas, 1994), and (5) response speed and list discrimination difficulty (Yonelinas \& Jacoby, 
1996). The results from these studies are summarized in Table 1. In all of these experiments, there is an easy or low-noise condition (a good learning task, capable subjects, short lists, ample study time, no speeded responding) and a difficult or high-noise condition (a poor learning task, less capable subjects, long lists, inadequate study time, speeded responding). With one minor exception in Table 1, relative to the easy condition, the difficult condition produces a decline in $I$ and an increase in $E$.

As is apparent in Table 1, the manipulations of list length and the time allowed to respond produced only small changes in $F$. In contrast, other manipulations produced a larger effect on $F$. Manipulations that produced little or no change in $F$ have been interpreted as supporting the assumption that $F$ is an estimate of the automatic component in dual-processing theory. In contrast, manipulations that produced a change in both $F$ and $R$ have been interpreted as producing parallel effects on the automatic and strategic components of dual-processing theory. Before accepting these interpretations, it is necessary to determine whether there are alternative explanations and whether there is sufficient power in the process dissociation procedure to discriminate among these alternative explanations.

\section{The Mirror Model}

Hirshman (1998) has pointed out that a negative correlation between $I$ and $E$ will produce approximately constant values of $F$ and that this is the pattern of results that one would expect if $I$ and $E$ are mirroring, as is typically the case in recognition memory (Glanzer \& Adams, 1990; McClelland \& Chappell, 1998). We would like to explore this point further by proposing a simple measurement model of the mirror effect and comparing the equations that result with those Jacoby (1991) derived to describe the contributions of familiarity and recollection in the process dissociation procedure. The ubiquity of the mirror effect is the reason for proposing that the mirror effect may apply to the process dissociation procedure. That is, because mirroring occurs with so many different experimental manipulations, it looks like mirroring may be the fundamental response of the decision mechanism to any increase in difficulty.

Our mirror model assumes that there is a bias point $(b)$ to which the hit rate and the false-alarm rate will converge as the ability to discriminate between correct and incorrect targets $(d)$ goes to zero. Furthermore, we assume that as $d$ increases, the hit and false-alarm rates move toward one and zero, respectively. The distance moved is in proportion to the distance from the bias point to the maximum or minimum score obtainable (1.0 and 0.0$)$. The latter assumption is likely to be approximately correct whenever the dependent variable is relative frequency, since it captures the intuition/generalization that an increase from .9 to .95 is psychologically more meaningful or larger than is an increase from .5 to .55 . For instance, suppose we have a conservative criterion $b=.30$. A discriminability of .5 would provide a hit rate of .65 (halfway to 1.0 ) and a false-alarm rate of .15 (halfway to zero). If discriminability remains the same and the bias changes to .5 , then the hit rate will be .75 and the false-alarm rate will be 25 . Given this model, we can write the following equations:

$$
\begin{gathered}
\text { hit rate }(\mathrm{HR})=b+d(1-b)=d+b-d b \\
\text { false-alarm rate }(\mathrm{FAR})=b-d b=b(1-d) .
\end{gathered}
$$

In applying this model to the process dissociation procedure, there are two primary forms of discriminability: old/new discriminability $\left(d_{\mathrm{O} / \mathrm{N}}\right)$ and list discriminability $\left(d_{\mathrm{L}}\right)$. In both Jacoby's (1991) and Yonelinas's (1994) form of the procedure, the response to completely new words is likely to depend primarily on old/new discriminability. Therefore, the false-alarm rate would be

$$
\mathrm{FAR}=b\left(1-d_{\mathrm{O} / \mathrm{N}}\right)
$$

In addition, in both procedures, exclusion rates are likely to depend primarily on list discrimination, especially under conditions in which old/new discriminability is good. Therefore, the exclusion rates will be

$$
\text { exclusion }(E)=b\left(1-d_{\mathrm{L}}\right) \text {. }
$$

For the inclusion rate, there is likely to be a difference between the Jacoby (1991) procedure and the Yonelinas (1994) procedure. In the Jacoby procedure, the inclusion condition is identical to normal recognition, and old/new discriminability is likely to dominate. Using this procedure, the inclusion rate would be

$$
\text { inclusion }(I)=d_{\mathrm{O} / \mathrm{N}}+b-d_{\mathrm{O} / \mathrm{N}} b \text {. }
$$

However, inclusion under the Yonelinas (1994) procedure is closer to a list discrimination judgment and given conditions where old/new discrimination is relatively good is likely to be dominated by list discriminability $\left(d_{\mathrm{L}}\right)$. Using this procedure, the inclusion rate would be

$$
\text { inclusion }(I)=d_{\mathrm{L}}+b-d_{\mathrm{L}} b \text {. }
$$

If we consider the Yonelinas (1994) procedure, in which the invariance of familiarity has been most striking, and we substitute $F$ for $b$ and $R$ for $d_{\mathrm{L}}$, we see that these equations are identical to the process dissociation equations. However, none of the theoretical development of these constructs refers to automatic or strategic constructs or to constructs that can be put in opposition. According to this account, an invariance of $F$ might reflect an invariance of $b$, the bias measure.

The mirror model makes an important prediction. Provided the Yonelinas (1994) procedure is employed, inclusion and exclusion rates are free to mirror, bias is not changing, and old/new discriminability is good, $F$ will be invariant regardless of changes in list discriminability. In the first and second experiments of Yonelinas (1994) and the first experiment of Yonelinas and Jacoby (1994), all of these conditions apply, and we see an invariance of $F$. By contrast, in experiments reported by Mulligan and Hirshman (1997), Yonelinas and Jacoby (1996), and Gruppuso 
Table 1

Selected Process Dissociation Experiments Showing Probabilities for Inclusion and Exclusion, False Alarms to New Words (New), and Estimates for Recollection $(R)$ and Familiarity $(F)$

\begin{tabular}{|c|c|c|c|c|c|}
\hline \multirow[b]{2}{*}{ Variable } & \multirow[b]{2}{*}{ Task Condition } & \multicolumn{2}{|c|}{$\begin{array}{c}\text { Probability of } \\
\text { Positive Response }\end{array}$} & \multicolumn{2}{|c|}{$\begin{array}{c}\text { Process } \\
\text { Dissociation Estimate }\end{array}$} \\
\hline & & Inclusion & Exclusion & $P(R)$ & $P(F)$ \\
\hline \multicolumn{6}{|c|}{ Jacoby (1991) Experiment 3} \\
\hline Generate vs. Read & $\begin{array}{l}\text { Anagram } \\
\text { Read } \\
\text { Heard } \\
\text { New }\end{array}$ & $\begin{array}{l}.80 \\
.48 \\
.69 \\
.18\end{array}$ & $\begin{array}{l}.29 \\
.37 \\
.67 \\
.22\end{array}$ & $\begin{array}{l}.51 \\
.11\end{array}$ & $\begin{array}{l}.59 \\
.42\end{array}$ \\
\hline \multicolumn{6}{|c|}{ Jennings and Jacoby (1993) Experiment 2} \\
\hline Age (Repetition at lag 12) & $\begin{array}{l}\text { Young } \\
\text { New } \\
\text { Old } \\
\text { New }\end{array}$ & $\begin{array}{l}.94 \\
.09 \\
.88 \\
.17\end{array}$ & $\begin{array}{l}.12 \\
.10 \\
.36 \\
.15\end{array}$ & $\begin{array}{l}.83 \\
.51\end{array}$ & $\begin{array}{l}.66 \\
.74\end{array}$ \\
\hline \multicolumn{6}{|c|}{ Yonelinas (1994) Experiment 1} \\
\hline List length & $\begin{array}{l}\text { Short } \\
\text { New } \\
\text { Long } \\
\text { New }\end{array}$ & $\begin{array}{l}.78 \\
.09 \\
.70 \\
.14\end{array}$ & $\begin{array}{l}.22 \\
.30\end{array}$ & $\begin{array}{l}.56 \\
.40\end{array}$ & $\begin{array}{l}.50 \\
.50\end{array}$ \\
\hline \multicolumn{6}{|c|}{ Yonelinas (1994) Experiment 2} \\
\hline List length & $\begin{array}{l}\text { Short } \\
\text { New } \\
\text { Long } \\
\text { New }\end{array}$ & $\begin{array}{l}.79 \\
.04 \\
.69 \\
.11\end{array}$ & $\begin{array}{l}.17 \\
.30\end{array}$ & $\begin{array}{l}.62 \\
.39\end{array}$ & $\begin{array}{l}.45 \\
.49\end{array}$ \\
\hline \multicolumn{6}{|c|}{ Yonelinas (1994) Experiment 3} \\
\hline Duration of study & $\begin{array}{l}3 \mathrm{sec} \\
1 \mathrm{sec} \\
\text { New }\end{array}$ & $\begin{array}{l}.64 \\
.51 \\
.24\end{array}$ & $\begin{array}{l}.42 \\
.40\end{array}$ & $\begin{array}{l}.22 \\
.10\end{array}$ & $\begin{array}{l}.54 \\
.45\end{array}$ \\
\hline \multicolumn{6}{|c|}{ Yonelinas and Jacoby (1994) Experiment 1} \\
\hline List length & $\begin{array}{l}\text { Short } \\
\text { New } \\
\text { Long } \\
\text { New }\end{array}$ & $\begin{array}{l}.58 \\
.18 \\
.50 \\
.20\end{array}$ & $\begin{array}{l}.23 \\
.25\end{array}$ & $\begin{array}{l}.35 \\
.25\end{array}$ & $\begin{array}{l}.35 \\
.33\end{array}$ \\
\hline \multicolumn{6}{|c|}{ Yonelinas and Jacoby (1996) } \\
\hline $\begin{array}{l}\text { Speeded responding and } \\
\text { discrimination difficulty }\end{array}$ & $\begin{array}{c}\text { Easy } \\
\text { Slow } \\
\text { New } \\
\text { Fast } \\
\text { New }\end{array}$ & $\begin{array}{l}.78 \\
.29 \\
.68 \\
.34\end{array}$ & $\begin{array}{l}.28 \\
.44\end{array}$ & $\begin{array}{l}.50 \\
.24\end{array}$ & $\begin{array}{l}.56 \\
.57\end{array}$ \\
\hline & $\begin{array}{c}\text { Hard } \\
\text { Slow } \\
\text { New } \\
\text { Fast } \\
\text { New }\end{array}$ & $\begin{array}{l}.74 \\
.30 \\
.66 \\
.31 \\
\end{array}$ & $\begin{array}{l}.55 \\
.62\end{array}$ & $\begin{array}{l}.18 \\
.04\end{array}$ & $\begin{array}{l}.67 \\
.64\end{array}$ \\
\hline
\end{tabular}

et al. (1997), manipulations of list discriminability resulted in changes in the estimate of $F$. In the first case, however, the Jacoby (1991) procedure was employed, so the inclusion rate should have been dominated by old/ new disciminability, and old/new discriminability does not change with the discriminability manipulation. In the second case, old/new discriminability was poor, but the instructions emphasized old/new discriminability, so the usual dominance of list discriminability in this paradigm may not have occurred. In the third case, the Yonelinas procedure was employed, but the inclusion and exclusion rates were not free to mirror. Subjects were given two lists. For each item on the first list, they either estimated the monetary value of an item or estimated its frequency. On the second list, half the participants were asked to estimate monetary value for all of the items, and the other half were asked to estimate the frequency for all of the items. At test, they were asked to include List 2 items and exclude List 1 items, so that inclusion and exclusion probabilities were collected within subjects. As Gruppuso et al. pointed out, the use of the single-test procedure eliminates the possibility of criterion shifts between inclusion and exclusion tests. In addition, the design eliminates the possibility of criterion shifts (or dis- 
$1 \mathrm{~A}$

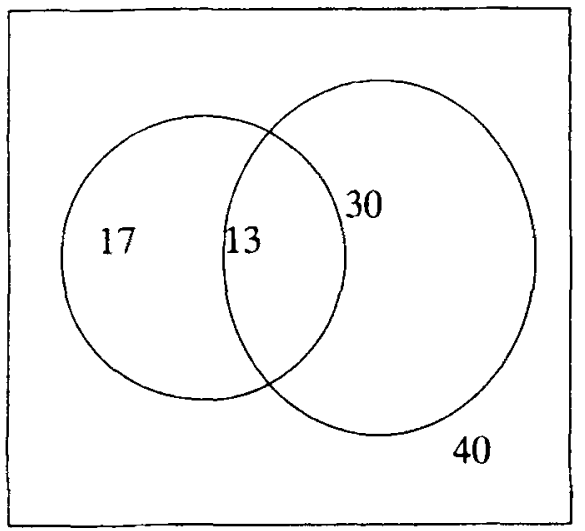

$1 \mathrm{C}$

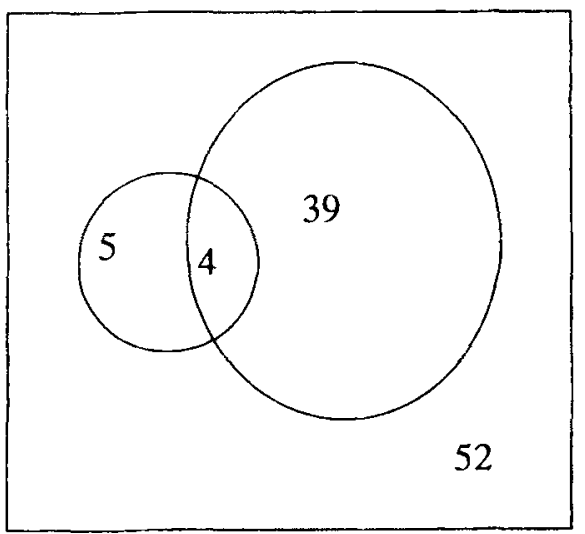

$1 \mathrm{~B}$

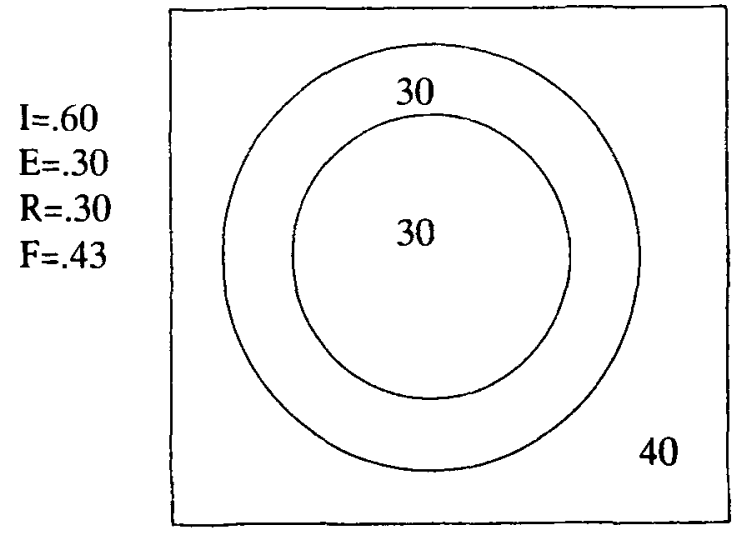

1D

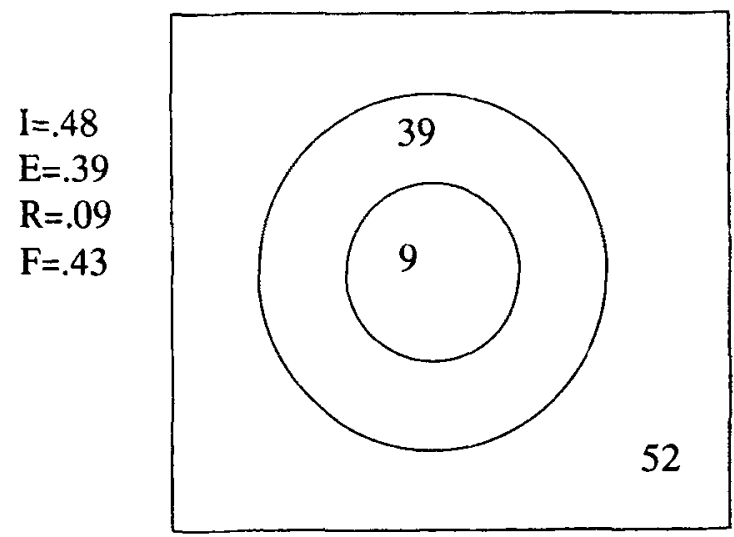

Figure 1. Venn diagrams detailing changes from an easy condition to a difficult condition under independence and redundancy assumptions. The panels on the left illustrate the independence assumption, and those on the right illustrate the redundancy assumption. The top panels illustrate the easy condition, and the bottom panels illustrate the difficult condition.

tribution shifts) between the low- and high-similarity conditions and, hence, eliminates the possibility of mirroring. In summary, it seems that the mirror model provides an alternative conception of the data produced by the process dissociation procedure that requires few assumptions about the underlying mechanism beyond the empirical observations that hit rates and false-alarm rates tend to mirror as discriminability is compromised.

\section{Independence Versus Redundancy}

As we have indicated earlier, Humphreys and Bain (1983) were able to show that the data presented by Mander (1980) either did not or could not, in principle, distinguish between independence (the assumption that two types of information or two processes are independent) and redundancy (the assumption that one type of information or process depends on the other). In the next three sections, we first address the issue of whether an assumption involving redundancy could produce a rough invariance in $F$, we then address the question as to whether the process dissociation procedure has the power to discrim- inate between independence and redundancy, and, finally, we address the issue as to why it is so difficult to determine the relationship between the processes/information involved in recognition.

In Figure 1, we present four Venn diagrams depicting the changes from an easy condition to a difficult condition assuming both independence and redundancy. In discussing these diagrams, we will interpret independence as an assumption about the relationship between processes and redundancy as an assumption about the relationship between information. This equation of independence with process and redundancy with information is not strictly necessary but is in agreement with the general trend in the literature.

The diagrams on the left of Figure 1 represent the independence assumption. For example, in Figure 1A, there are 17 list items for which recollection is successful but for which there is no familiarity, 30 list items for which there is familiarity but no recollection, and 13 list items for which there is both familiarity and recollection. Figure $1 \mathrm{~A}$ represents performance in the easy condition, and 


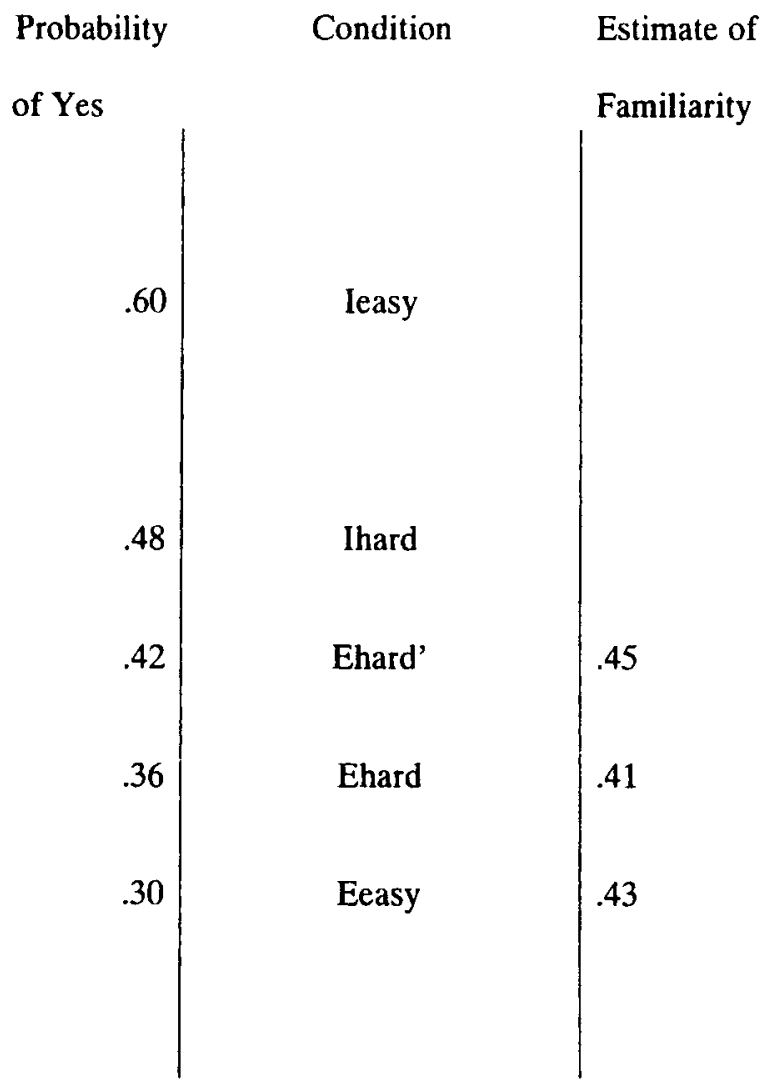

Figure 2. Ieasy and $E$ easy are the obtained values for $I$ and $E$ in the easy condition, and $I$ hard and $E$ hard are the obtained values for $I$ and $E$ in the hard condition. Ehard' is a value of $E$ that would have produced an estimate of $F$ as close to the value obtained in the easy condition as was produced by the obtained value ( $E$ hard). The calculation of $F$ assumes that the values for $I$ are fixed for their respective conditions and are shown next to the corresponding $E$ values.

Figure $1 \mathrm{C}$ represents performance in the difficult condition. The panels on the right represent the redundancy assumption. Under this assumption, the subject has access to both list and item information (information that allows the correct assignment of items to the list and to the experiment, respectively) for items in the inner circle ( 30 in Figure 1B) and has only item information for items in the outer ring ( 30 in Figure 1B). Figure 1B represents the easy condition, and Figure $1 \mathrm{D}$ represents the difficult condition. The values for $I, E, R$, and $F$ implied by the diagrams in the panels are presented in the body of the figure. To two decimal places, the values for $I, E, R$, and $F$ are the same for the independence and redundancy models. Thus, both the independence model and the redundancy model show a change in $R$ and an invariance in $F$ as we go from the easy condition to the difficult condition. The pattern that produces this invariancy for the redundancy model involves a rise in the number of items in the outer ring and outside the circles, with a fall in the number of items in the inner circle.
Mulligan and Hirshman (1997; also see Humphreys, 1976, and Humphreys \& Bain, 1983) have interpreted the redundancy relationship depicted in the right panels of Figure 1. Mulligan and Hirshman postulate that two types of information - diagnostic information that differentiates between lists and between old and new items (the inner circle) and nondiagnostic information that only differentiates between old and new items (the outer ring) - are involved.

By expanding slightly on the Mulligan and Hirshman (1997) diagnostic context model, it is possible to show how it could plausibly accommodate a rough invariance in $F$ for many experimental manipulations. In this version of the diagnostic context model, we reinterpret the Venn diagrams so that the sets in the diagrams no longer refer to items but refer to elements that can be sampled whenever an item is presented. That is, assume that the elements in the right panels of Figure 1 represent information that can be sampled on a trial. On each trial, exactly 1 out of the 100 elements is sampled. The sampled element differentiates between old and new items and between lists, differentiates between old and new items only, or does not differentiate.

Next, assume that the information used to differentiate between lists is "finer grained" than the information used to differentiate between old and new items. More precisely, assume that when noise (e.g., the subject performs a concurrent task at study) is added to the system, some elements that would have differentiated between lists as well as between old and new items will now differentiate only between old and new items and that some elements that would have differentiated only between old and new items will no longer differentiate. With these assumptions, it is plausible that, with the addition of a moderate amount of noise, there will be a decline in the number of elements that differentiate between both lists and old and new items, an increase in the number of elements that differentiate between old and new items but not lists, and an increase in the number of elements that do not differentiate. These changes will produce a rough invariance in $F$, and, as we show next, there does not appear to be enough power in the process dissociation procedure to differentiate between independence and redundancy.

\section{The Power to Discriminate Between Alternative Dual-Processing Models}

To examine the issue of power, we assume that the obtained values of $I$ and $E$ in the easy condition along with $I$ in the hard condition are fixed and then determine the realistic range over which $E$ in the hard condition can vary. For example, in Figure 2, it is reasonable to assume that $E$ in the hard condition could have ranged from .30 (the value of $E$ in the easy condition) to .48 (the value of $I$ in the hard condition). Now, assume that we had obtained a value for $E$ in the hard condition of .36 . This value of $E$ corresponds to a value of $F$ of .41 , which is only slightly lower than the value of .43 that was found in the easy condition. Since an obtained value for $E$ of .42 
corresponds to a value of $F$ of .45 , any value of $E$ in the hard condition between .36 and .42 will result in an estimate of $F$ as close or closer to the value in the easy condition as the obtained value of $F=.41$. In evaluating how likely such a finding is, we start with the assumption that all values of $E$ between .30 and .48 are equally likely. Under this assumption, the probability of finding a value of $F$ within .02 of the value in the easy condition is $.06 / .18=.33$. This probability would be even higher if, as seems likely, experimenters design studies so that values of $E$ in the difficult condition that are close to the value of $E$ in the easy condition or close to the value of $I$ in the difficult condition are unlikely.

Thus, under ideal conditions, where there are no complications resulting from bias (guessing changes), and from correlations between $F$ and $R$ induced by item or subject differences (Curran \& Hintzman, 1995), and there are no false recollections, it will be extremely difficult to discriminate between independence and redundancy. It will be even more difficult to discriminate between independence and redundancy where all of these factors will play some role.

\section{Why It Is So Difficult to Determine the Relationship Between the Factors}

The basic reason why it is so difficult to discriminate between independence and redundancy in the process dissociation procedure is that the two approaches make identical predictions about populations of items. That is, the redundancy assumption asserts that, ignoring guessing, an item cannot be assigned to its correct list unless it can be recognized. The independence assumption makes the same prediction about the relationship between list and old/new discrimination but for a different reason. That is, although successful recollection is assumed to be independent of familiarity, it also enables both correct list assignment and recognition. Thus, both the redundancy assumption and the independence assumption assert that there will be a set of items that can be correctly assigned to both the experiment and a list, a set of items that can be assigned to the experiment and not a list, and a set that can be assigned neither to the experiment nor to a list. Both approaches also predict that there will be no items that can be assigned to a list but not to the experiment.

The empirical parameters estimated by the process dissociation procedure are interpretable in terms of the proportion of list items that occur in these sets. That is, ignoring any complications due to guessing or response bias, $I-E$ is the probability of discriminating between lists, $E /(1-l+E)$ is the probability of discriminating between old and new words given that one cannot discriminate between lists, $I$ is the unconditional probability of being able to discriminate between old and new items, and $E$ is the joint probability of discriminating between old and new items and not discriminating between lists. This common interpretation in terms of the proportion of items contained in one of these sets is the reason that Humphreys and Bain (1983) were able to show that the in- dependence and redundancy assumptions were, in principle, indistinguishable in much of Mandler's (1980) data. In process dissociation data, they are distinguishable, in principle; however, in practice, it will be difficult, if not impossible.

Another example where the empirical probabilities drive the permissible interpretation of data and not the underlying dual-processing theory can be found in the study of Jacoby, Jones, and Dolan (1998), in which subjects read List 1 words, which were presented one, two, or three times, and heard List 2 words. In one condition, subjects were asked to state whether they were making an old decision because they could remember or because they just knew that the item was old. ${ }^{2}$ In the other condition, subjects were asked to say "yes" to List 2 (heard) items and "no" to List 1 (read) and new items.

Jacoby et al. (1998) argued that the probability of a "know" response $(K)$ to a List 1 item (the subject knows that it is old but does not remember or recollect) should equal the exclusion probability of falsely saying "yes" to a List 1 item (the item is familiar but the subject does not recollect that it occurred in List 1). The probabilities, in the exclusion condition, of saying "yes" to a once, twice, and thrice presented word were $.26, .25$, and .24 , respectively. The comparable probabilities of reporting that they simply knew that the item was old were $.34, .31$, and .29 , respectively. There was no significant difference in these results as a function of either number of presentations or type of response (exclusion false alarm vs. know).

These results are potentially important. ${ }^{3}$ They indicate that there is a reasonably close relationship between the probability of discriminating between old and new items but not discriminating between lists $(E)$ and the probability that a subject will know that an item is old but not remember it $(K)$. On the basis of this correspondence between $E$ and $K$, one has support for the conclusion that the information that evokes a "remember" response also permits list discrimination, and vice versa.

This correspondence between $E$ and $K$ also provides support for the assumption that two types of information are involved. That is, if the information that evokes a "remember" response ałso underlies list discrimination, there would be a qualitative, not just a quantitative, difference between the information that supports "remember" and "know" responses. Thus, one could reject the Donaldson (1996) and Hirshman and Master (1997) proposal that a two-criteria signal detection model is an adequate explanation for remember-know judgments.

There is, however, no basis in the correspondence between $E$ and $K$ to infer anything about the relationship between the information/processes that underlie these responses. That is, the crucial assumption is that the information that evokes a "remember" response is equivalent to the information that leads to successful list discrimination. If this assumption is accepted, then the independence model (Jacoby, 1991), the redundancy model (Humphreys \& Bain, 1983; Mulligan \& Hirshman, 1997), and the exclusivity model (Gardiner \& Parkin, 
1990) all predict that there will be a set of items that will be recognized, will evoke a "know" response, and will not lead to successful list discrimination; there will be another set of items that will be recognized, will evoke a "remember" response, and will lead to successful list discrimination; and there will be a third set of items that will not be recognized, will evoke neither a "know" nor a "remember" response, and will not lead to successful list discrimination. Thus, the observation that $E=K$ cannot, in principle, discriminate between the independence, redundancy, and exclusivity dual-processing models.

\section{Other Complications/Problems \\ With the Process Dissociation Procedure}

In this section, we briefly discuss problems with using historical data to support current concepts, and, then, we address Jacoby's (1991) attempt to provide converging evidence on what the process dissociation parameter, $F$, estimates. We then consider two examples where limitations on theoretical thinking are imposed by the lack of details about representation and process. These examples are the extension of the process dissociation procedure to cued recall/stem completion (Jacoby, Toth, \& Yonelinas, 1993 ) and the reaction of Yonelinas and Jacoby (1996) to an apparent disconfirmation of the process dissociation procedure. Finally, we consider the implications of being able to correlate self-reports about subjective states or inclusion/exclusion judgments with brain activity.

As we indicated in the introduction, many different paradigms have been interpreted as supporting dual processes in recognition. However, there are many unanswered questions about these paradigms (see Bain \& Humphreys, 1988, and Humphreys \& Bain, 1983, for examples), and it is not always clear that this earlier evidence would stand up to contemporary scrutiny. For example, Atkinson and Juola's (1974) evidence for dual processes had primarily consisted of a demonstration that mean reaction times could be decomposed into two hypothetical processes. We do not know whether their interpretation would stand up to a contemporary analysis in which reaction time distributions, not just reaction time means, were examined.

Jacoby's (1991) procedure for providing converging evidence on the parameters estimated by the process dissociation procedure also encounters some difficulties. That is, Jacoby compared estimates of $F$ using the process dissociation procedure with exclusion performance under divided attention conditions. When these procedures produce comparable estimates, they provide some support for the assumption that $F$, as estimated by the process dissociation procedure, is "automatic" and also provide support for the process dissociation procedure. However, any assumption of automaticity comes with boundary conditions, and these boundary conditions are poorly specified in dual-processing theory. For example, if the divided attention task becomes too demanding (e.g., on some trials, a subject does not pay attention to the target), then the theoretical construct of familiarity must be impaired.
Thus, the only prediction that can be made from the automaticity assumption is that performance under some level or range of levels of divided attention will correspond to the estimate of $F$ obtained from the process dissociation procedure.

The lack of detail about representation and process also makes it difficult to determine whether extensions to theoretical thinking are reasonable. For example, Jacoby et al. (1993) applied the process dissociation procedure to cued recall/stem completion. In this new application, they provided subjects with a stem cue and asked them either to complete the stem with a list word and, if they could not think of a list word, to complete it with the first word that came to mind (inclusion) or to complete the stem with any word other than a list word (exclusion). This application of the procedure also produces estimates of two constructs that are frequently referred to as conscious and unconscious influences on memory. Furthermore, this procedure allows direct comparisons to be made between the estimate of the unconscious influence and performance on an indirect test (complete this stem with the first word that comes to mind). Because of the similarity in the terminology used and the use of a common set of historical references, it is seldom clear whether or not comments on the automatic component estimated from the process dissociation procedure applied to cued recall/stem completion also apply to the process dissociation procedure's estimate of familiarity. There is certainly a resemblance between the two sets of constructs (conscious and unconscious vs. recollection and familiarity), but whether this is just a surface similarity or a structural correspondence cannot be answered in a theory that eschews most reference to structure.

Another example, in which a lack of theoretical detail makes it very difficult to determine whether a theoretical extension is reasonable, can be found in Yonelinas and Jacoby's (1996) reaction to an apparent disconfirmation of the process dissociation procedure. They presented subjects with words that appeared either on the left-hand side of the screen or the right-hand side of the screen, in either a large font or a small font. The instructions focused the subjects' attention on the side on which each word appeared by asking them to associate the words on the left with one distinctive person or place and the words on the right with another person or place so as to aid later recall (intentional condition). By contrast, the instructions suggested that the manipulation of font size was designed to change reading difficulty (incidental condition). At test, subjects were required to say "yes" to words that appeared on one side and "no" to words from the other side and new words (easy discrimination), or they were required to say "yes" to words that appeared in one font size and "no" to words of the other size and new words (hard discrimination). In addition, they required subjects to respond either quickly or slowly. There were two important findings (see Table 1). First, as discrimination difficulty increased, estimates of $F$ increased and estimates of $R$ decreased. Second, speeded responding had no discernable impact on 
estimates of $F$, though it substantially reduced estimates of $R$.

Yonelinas and Jacoby (1996) interpreted these results as requiring a distinction between criterial and noncriterial recollection. That is, recollection of the screen side would be criterial when subjects were instructed to discriminate on the basis of screen side, but it would be noncriterial when subjects were instructed to discriminate on the basis of font. Furthermore, they assumed that the presence of noncriterial recollection would inflate the estimate of $F$. At this point, they had basically two options to explain why an estimate of $F$ that was inflated by noncriteral recollection was not affected by a requirement to respond quickly. They could have retained the assumption that recollection was strategic and therefore should have been affected by a requirement for speeded processing. This option would have led them to reject the process dissociation procedure. Instead, they opted for the conclusion that noncriterial recollection, like familiarity, was automatic and therefore was not affected by the speeded processing requirement. That is, they opted to support the process dissociation procedure over their prior assumption about recollection. Such a decision may be reasonable given the authors understanding of the existing data and/or their implicit assumptions about the nature of recollection and familiarity. However, it is almost impossible for an outsider, someone who does not share their understanding of the existing data and/or their implicit assumptions, to evaluate the reasonableness of their decision.

As a final point, there has been considerable interest in the possibility of being able to correlate either self-reports about subjective states or inclusion/exclusion judgments with brain activity (cf. Duzel, Yonelinas, Mangun, Heinze, \& Tulving, 1997). However, finding a correlation is not going to provide a simple means to validate dual-process theories. In particular, a finding of differential brain activity does not imply separate processes, nor does it imply that the brain activity causes the response. For example, the right frontal activity that accompanies "remember" responses and correct source monitoring decisions (Duzel et al., 1997; Wilding \& Rugg, 1996, 1997) may be a sequela to trace retrieval and the recognition decision but not a cause of the decision. Nevertheless, the ability to determine how patterns of brain activity covary with experimental manipulations may help us resolve some of the seemingly intractable problems faced by dual-processing theories of recognition. One of the first problems that could be addressed is whether the activity that occurs in the process dissociation procedure or in the remember/ know procedure also occurs in yes/no recognition. That is, is the evidence for a recall-like process in recognition induced by the special paradigms we use, or is it more generally a component of our standard paradigms?

\section{Conclusions}

Dual-processing explanations for the similarities and differences between recognition and recall paradigms started by focusing on organizational effects in recognition, shifted to the hypothetical role played by perceptual fluency, and then shifted again to the process dissociation procedure. This history might be seen as an indicator of cumulative progress. In our opinion, however, progress has been limited. At the present time, we can reject a single-factor model for recognition since it seems clear that two factors (processes, information, or cues) are involved in at least some recognition decisions. However, we have not been able to go beyond the rejection of a singlefactor model and to identify the relationship between the factors and determine whether fundamentally different processes are involved. That is, in 1983, Humphreys and Bain were able to show that the evidence available at that time did not or could not distinguish between alternative dual-processing theories (in particular, independence vs. redundancy). In this paper, we have shown that the dualprocessing procedure has not and probably cannot resolve this issue.

In our opinion, the key to resolving this problem is to incorporate more content (assumptions about representations and the processes that operate on those representations) into dual-processing theory. Brainerd, Reyna, and Mojardin (1999) provide one example of this approach using a measurement model. As they have indicated, it is not possible to test Jacoby's (1991) dual-processing theory by providing a global measure of goodness of fit or to test alternative hypotheses. The problem is that there are two data points, $E$ and $I$, and two parameters, $R$ and $F$, so there are no degrees of freedom left over. Brainerd et al.'s solution was to add enough content to make the model testable. That is, the additional content permits new predictions so that there are more predictions than parameters.

Fully fledged process models (e.g., Dennis \& Humphreys, in press; Ratcliff, Van Zandt, \& McKoon, 1995) have also been proposed to explain results that have been interpreted as supporting specific versions of dualprocessing theory. These models are wrong in the sense that all formal models are wrong. That is, with all formal models, there will be some systematic discrepancies between theory and data. In addition, formal models must be treated as an example of a class of permissable models, but it is difficult to specify the class. They have, however, a major advantage over dual-processing theory as it has developed. That is, when concepts and/or the relationship between constructs are made explicit, at times it can become possible to reject constructs outright (e.g., see Clark \& Gronlund's, 1996, discussion of the impact of the null list strength finding). At other times, it is not possible to reject a concept outright, but the model becomes cumbersome, and a newer theory can clearly be shown to provide a more parsimonious, or more general, explanation.

\section{REFERENCES}

Atkinson, R. C., \& Juola, J. F. (1974). Search and decision processes in recognition memory. In D. H. Krantz, R. C. Atkinson, R. D. Luce, 
\& P. Suppes (Eds.), Contemporary developments in mathematical psychology: Vol. l. Learning, memory, and thinking (pp. 243-293). San Francisco: Freeman.

BaIN, J. D., \& HumphreYs, M. S. (1988). The relational context effect: Cues, meanings or configurations. In G. Davies \& D. Thomson (Eds.), Memory in context: Context in memory (pp. 97-138). London: Wiley.

Brainerd, C. J., Reyna, V. F., \& Mojardin, A. H. (1999). Conjoint recognition. Psychological Review, 106, 160-179.

Chalmers, K. A., \& Humphreys, M. S. (1998). Role of generalized and episode specific memories in the word frequency effect in recognition. Journal of Experimental Psychology: Learning, Memory, \& Cognition, 24, 610-632.

Clark, S. E., \& Gronlund, S. D. (1996). Global matching models of recognition memory: How the models match the data. Psychonomic Bulletin \& Review, 3, 37-60.

Curran, T., \& Hintzman, D. L. (1995). Violations of the independence assumption in process dissociation. Journal of Experimental Psychology: Learning, Memory, \& Cognition, 21, 531-547.

DENNIS, S., \& HumPHREYS, M. S. (in press). A context noise model for episodic recognition. Psychological Review.

Dodson, C. S., \& Johnson, M. K. (1996). Some problems with the process dissociation approach to memory. Journal of Experimental Psy. chology: General, 125, 181-194.

Donaldson, W. (1996). The role of decision processes in remembering and knowing. Memory \& Cognition, 24, 523-533.

Duzel, E., Yonelinas, A. P., Mangun, G. R., Heinze, H.-J., \& TulVING, E. (1997). Event-related brain potential correlates of two states of conscious awareness in memory. Proceedings of the National Academy of Sciences, 94, 5973-5978.

GARDINER, J. M. (1988). Functional aspects of recollective experience. Memory \& Cognition, 16, 309-313.

Gardiner, J. M., \& PARKIN, A. J. (1990). Attention and recollective experience in recognition memory. Memory \& Cognition, 18, 579-583.

Gardiner, J. M., Richardson-Klavehn, A., \& Ramponi, C. (1997). On reporting recollective experiences and "direct access to memory systems." Psychological Science, 8, 391-394.

GlanZER, M., \& AdAMS, J. K. (1990). The mirror effect in recognition memory: Data and theory. Journal of Experimental Psychology: Learning, Memory, \& Cognition, 16, 5-16.

Gruppuso, V., Lindsay, D. S., \& Kelly, C. M. (1997). The processdissociation procedure and similarity: Defining and estimating recollection and familiarity in recognition memory. Journal of Experimental Psychology: Learning, Memory, \& Cognition, 23, 259-278.

Hirshman, E. (1998). On the logic of testing the independence assumption in the process-dissociation procedure. Memory \& Cognition, 26, 857-859.

Hirshman, E., \& Master, S. (1997). Modeling the conscious correlates of recognition memory: Reflections on the remember-know paradigm. Memory \& Cognition, 25, 345-351.

HuMPHREYS, M. S. (1976). Relational information and the context effect in recognition memory. Memory \& Cognition, 4, 221-232.

HumphreYs, M. S., \& Bain, J. D. (1983). Recognition memory: A cue and information analysis. Memory \& Cognition, 11, 583-600.

JACOBY, L. L. (1991). A process dissociation framework: Separating automatic from intentional uses of memory. Journal of Memory \& Language, 30, 513-541.

JACOBY, L. L., \& DaLlas, M. (1981). On the relationship between autobiographical memory and perceptual learning. Journal of Experimental Psychology: General, 110, 306-340.

JACOBY, L. L., JONES, T. C., \& DOLAN, P. O. (1998). Two effects of repetition: Support for a dual-process model of know judgments and exclusion errors. Psychonomic Bulletin \& Review, 5, 705-709.

Jacoby, L. L., Toth, J. P., \& Yonelinas, A. P. (1993). Separating conscious and unconscious influences of memory: Measuring recollection. Journal of Experimental Psychology: General, 123, 216-219.

JAMEs, W. (1890). Principles of psychology (Vol. 1). New York: Holt. JENnings, J. M., \& JACOBY, L. L. (1993). Automatic versus intentional uses of memory: Aging, attention, and control. Psychology \& Aging, 8, 283-293.

KINTSCH, W. (1970). Models for free recall and recognition. In D. A. Norman (Ed.), Models of human memory (pp. 331-373). New York: Academic Press.

KNOwLton, B. J. (1998). The relationship between remembering and knowing: A cognitive neuroscience perspective. Acta Psychologica, 98, 253-265

MANDLER, G. (1972). Organization and recognition. In E. Tulving \& W. Donaldson (Eds.), Organization of memory (pp. 139-166). New York: Academic Press.

MandLeR, G. (1980). Recognizing: The judgment of previous occurrence. Psychological Review, 87, 252-271.

McClelland, J. L., \& Chappell, M. (1998). Familiarity breeds differentiation: A subjective-likelihood approach to the effects of experience in recognition memory. Psychological Review, 105, 724-760.

MCDOUgaLL, R. (1904). Recognition and recall. Journal of Philosophical Psychology \& Scientific Method, 1, 229-233.

Mulligan, N. W., \& Hirshman, E. (1997). Measuring the bases of recognition memory: An investigation of the process-dissociation framework. Journal of Experimental Psychology: Learning, Memory, \& Cognition, 23, 280-304.

PoldRACK, R. A., \& Logan, G. (1997). Fluency and response speed in recognition judgments. Memory \& Cognition, 25, 1-10.

RatCliff, R., Van Zandt, T., \& MCKoon, G. (1995). Process dissociation, single-process theories, and recognition memory. Journal of Experimental Psychology: General, 124, 352-374.

Tulving, E. (1985). Memory and consciousness. Canadian Psychologist, 26, 1-12.

WILDING, E. L., \& RugG, M. D. (1996). An event-related potential study of recognition memory with and without retrieval of source. Brain, 119, 889-905.

WILDING, E. L., \& RUGG, M. D. (1997). Event-related potentials and the recognition exclusion task. Neuropsychologia, 35, 1185-1195. YoNELINAS, A. P. (1994). Receiver-operating characteristics in recognition memory: Evidence for a dual-process model. Journal of Experimental Psychology: Learning, Memory, \& Cognition, 20, 1341 1354.

Yonelinas, A. P., \& JACOBY, L. L. (1994). Dissociations of processes in recognition memory: Effects of interference and response speed. Canadian Journal of Experimental Psychology, 8, 516-534

Yonelinas, A. P., \& JaCoby, L. L. (1996). Noncriterial recollection: Familiarity as automatic, irrelevant recollection. Consciousness \& Cognition, 5, 131-141.

\section{NOTES}

1. Assumptions about representation and process come in varying degrees of specificity. For example, a distinction between item and relational/associative information contains a minimal commitment to the complexity of the binding employed, and a statement about the cues used is a start toward specifying the processes involved. One can also have a commitment to generic forms of representation and process, such as separate versus composite storage and sequential search versus direct access. In a fully fledged process model, the modeler specifies a precise form of representation and specific processes that operate on that representation. For example, words may be represented as vectors, memory as the sum of the vectors that correspond to the items in the list, and the memory access process as the dot product between the vector representing the target and memory. In all such models, there is an intention, though it is not always explicitly stated, that the model generalize across irrelevant details to a class of permissible models.

2 . Proponents of the remember/know procedure generally seem committed to the same set of ideas about dual processing as the proponents of the process dissociation procedure, and they also focus on measurement operations. The basic measurement operation in the remember/ know procedure is a self-report. That is, subjects are asked to report on whether they are identifying an item as old because they remember de- 
tails about the items presentation or because they simply "know" that it is old (Gardiner, 1988; Tulving, 1985). The central claim about this approach is that the two responses index or are associated with qualitatively different states of awareness. A less central but considerably more provocative claim is that the "know" response indexes a state of awareness that is associated with semantic/procedural memory and the "remember" response indexes a state of awareness associated with episodic/declarative memory (Gardiner, 1988). There is also a claim (Gardiner \& Parkin, $1990)$ that the relationship between "remember" and "know" responses is one of exclusivity. In a sense, this claim about exclusivity is necessarily correct since subjects are not allowed to report that they both remember and know that an item was old. However, the claim of exclusivity goes beyond this response incompatibility and is treated either as a statement about exclusive states of consciousness (Gardiner, RichardsonKlavehn, \& Ramponi, 1997) or as the basis to make predictions about how "know" and "remember" responses should change as a function of an experimental manipulation (Gardiner \& Parkin, 1990).

3. There is a conflict between Yonelinas and Jacoby's (1996) explanation for changes in $F$ as a function of the discriminability between two lists or sets of items and Jacoby et al.'s (1998) finding of a correspondence between $E$ and $K$ (also see Gruppuso et al., 1997, and Mulligan \& Hirshman, 1997). That is, in our laboratory, when subjects are asked to explain why they report remembering an item, they indicate that the recall of other list items and the recall of idiosyncratic responses to the study word are involved. However, according to Yonelinas and Jacoby (1996), these aspects of remembering or recollection should be noncriterial because they would not ordinarily discriminate between lists. Thus, the existence of noncriterial recollection should mean that $K$ is systematically larger than $E$. However, if there is no systematic difference between $E$ and $K$, it may indicate that when there is a very obvious distinction between lists or items (e.g., read vs. heard), subjects will focus almost entirely on that distinction. This focusing would have implications for our understanding of the nature of recollection. For example, does the output of the recollection process depend on what the subject expects to find? It may also indicate that recollection when a relatively homogenous set of items is involved may be different from recollection when two distinct types of items are involved. There is even the possibility that recollection is relatively unimportant with a homogenous set and becomes important only when subjects are asked to make "remember" and "know" judgments or to discriminate between two distinct types of items.

(Manuscript received April 4, 1999; revision accepted for publication January 13,2000 .) 